\title{
La investigación pedagógica y la metodología ABI percibida por los estudiantes de
} una Universidad Privada

\section{The pedagogical research and the ABI methodology perceived by the students of a Private University}

\author{
Ronald Alarcón ${ }^{1}$, Heyden Flores ${ }^{2}$
}

\section{RESUMEN}

El presente trabajo de investigación trata del abordaje de la didáctica basada en la investigación y como es percibida por los estudiantes de formación magisterial. Dentro del plano de los aprendizajes situados, el aprendizaje basado en la investigación es uno de los más fecundos que se puede utilizar en la formación docente, permite que el futuro docente desarrolle habilidades para reflexionar sobre su labor, identificar problemas y proponer una intervención que terminará en una nueva práctica denominada praxis ergo nueva teoría. El objeto de estudio es indagar sobre la aplicación del aprendizaje basado en la investigación y como se desarrolla al interior de las aulas, en tanto que el mismo parte de la formulación adecuada de un problema, siendo ello compatible con el proceso de problematización, ya que se parte de la realidad, de la experiencia obtenida en la práctica pedagógica a partir de lo cual se realiza una construcción critico reflexiva del aprendizaje siendo el detonante para partir con la investigación ya que el estudiante siente curiosidad por indagar sobre el problema que no lo deja avanzar en su quehacer docente.

Palabra Clave: Pedagogía, pensamiento crítico, didáctica, investigación formativa, metodologías educativas

\section{ABSTRACT}

This research work deals with the approach of didactics based on research and how it is perceived by teachers training students. Within the framework of situated learning, research-based learning is one of the most fruitful that can be used in teacher training, it allows future teachers to develop skills to reflect on their work, identify problems and propose an intervention that will end in a new practice called praxis ergo new theory. The object of study is to inquire about the application of research-based learning and how it is carried out within classrooms, while it starts from the proper formulation of a problem, being compatible with the problem-solving process, since it starts from reality, from the experience obtained in the pedagogical practice from which a critical and reflective construction of learning is carried out, being the trigger to start with the investigation since the student is curious to inquire about the problem that does not leave him advance in your teaching.

Keywords: Pedagogy, critical thinking, didactics, formative research, educational methodologies.

1. Dr. Ronald Jesús Alarcón Anco. .Educador y administrador, Maestro en Filosofía, y doctor en Administración. Docente UAP

2. Dr. Heyden Neil Flores De La Cruz. Educador y abogado. Maestro en filosofía y Doctor en Educación. Docente UAP 


\section{INTRODUCCIÓN}

Cuando se trata del abordaje pedagógico de la didáctica de la investigación se debe recabar información de cómo se dan los procesos pedagógicos, ello implica obtener información de la fuente primaria, el docente mismo en la acción reflexiva sobre su práctica pedagógica, en ese sentido para Sabino (1992), se define a la investigación como el acto de emprender una acción para resolver el problema desde la perspectiva del conocimiento (pág. 47), lo que significa hacer ciencia a partir de la ciencia.

La situación referida al emprendimiento de una acción para solucionar un problema posee una dimensión amplia cuando se aplica a la práctica pedagógica, los docentes cuando proponen un desafío a sus estudiantes a través de un caso extraído de la realidad(práctica pedagógica) y muestran la metodología para problematizar y formular la pregunta de investigación dan un primer paso para construir ciencia, muestran al estudiante como buscar información de fuentes primarias y utilizarla como sustento de las variables que intervienen en dicho problema, esta búsqueda los conduce a la definición conceptual de las variables y extracción de las dimensiones. Para tal fin, el estudiante parte de la exploración y consolida su acercamiento con el fenómeno pedagógico en el nivel descriptivo de investigación, ello le permite descubrir las propiedades intrínsecas del fenómeno estudiado permitiendo, en base a ello, diseñar estrategias metodológicas que mejoren su práctica pedagógica originado una praxis critica que regula su formación y cimenta el logro del perfil deseado.

Como se aprecia, en parte del planteamiento metodológico subyace la búsqueda de información, lo cual conducirá a fundamentar el acto problematizador y la fundamentación de las variables, ello conduce al estudiante a un primer contacto con la estructuración de las bases teórico científicas que han de sostener su hipótesis, la misma que en su calidad de posible respuesta al problema y guía de la investigación, constituye una ge- neralización, que siguiendo la ruta del método deductivo permitirá al estudiante ir descubriendo en forma reflexiva el camino para llegar a la validación de dicha hipótesis en un contexto empírico.

Retomando la metodología de aplicación del ABI, una vez tomada la decisión del tema a investigar, formulado el problema e informado del protocolo a seguir, se debe de monitorear en forma constante el avance de la ejecución de la investigación, ello es compatible con la gestión del acompañamiento para el logro de competencias y el producto final es sometido al proceso de evaluación.

La Investigación se realizó en la Escuela Profesional de Ciencias del Deporte de la Universidad Alas Peruanas" institución de carácter privado, la metodología empleada fue deductiva con un diseño de índole descriptivo, con enfoque cuantitativo, ontológicamente realista fundamentado en el neopositivismo. Por ello se seleccionaron 91 estudiantes de la especialidad de Ciencias del Deporte y 15 docentes haciendo un total de 106 sujetos de la muestra, quienes fueron sometidos a dos cuestionarios uno de ellos referido a la variable pedagogía de la investigación y otra variable sobre la construcción del saber, obteniéndose resultados interpretativos del desarrollo del ABI.

Así mismo se realizó análisis cualitativo de relatos obtenidos de entrevistas en profundidad practicado a 15 docentes de la facultad de ciencias del deporte, en el cual se describe la forma del abordaje del ABI por parte de los docentes, varios de ellos coincidieron en el siguiente relato, en síntesis se inicia la sesión de aprendizaje exponiendo el caso: “...dos personas se cruzan en la calle por casualidad, y se preguntan ¿Cómo te llamas?, esto sucede debido al insuficiente conocimiento del uno por el otro, están explorándose, así pueden continuar interrogándose hasta que, después de un periodo medio, ya se identifican y alguien le pregunta, a uno de ellos por el otro, ¿Cómo es tu compañero?, respondiendo el interrogado, es alegre o triste, estudioso o no, alto o 
bajo, es decir es capaz de describir sus características, lo que conduce a preguntar ¿entonces es necesario tener conocimiento previo para describir un fenómeno?, la respuesta es afirmativa, toda vez que la investigación descriptiva aplicada fue necesariamente de carácter ex post facto."

El proceso de aprendizaje basado en la investigación(ABI) es el camino para concretizar el desarrollo de las competencias referidas a la crítica reflexión que debe primar en el docente, para ello se parte de la formulación del problema de investigación, lo cual se colige con el proceso de problematización partiendo de la realidad, de la experiencia obtenida en la práctica pedagógica, para ello se informa a los estudiantes del protocolo a seguir, lo que es compatible con la gestión del acompañamiento para el logro de competencias partiendo desde la complejidad del pensamiento, para finalmente obtener un producto que es sometido a evaluación, recuérdese que, "El desarrollo de trabajos de investigación promueve la producción intelectual, lo que permite el ejercicio, y por lo tanto, el desarrollo de Pensamiento Crítico" (Mendoza, 2015, pág. 66).

Cuando se realiza el abordaje de la investigación, en materia de ciencias ligadas al deporte se debe tener especial cuidado en concebirla no como un simple acto de medición de sus variables, sino como el resultado de un proceso de interacción entre los miembros del colectivo que se dedica a la misma, toda vez que los resultados no solo linden con la medicina sino más se orientan al desarrollo del conocimiento como construcción social, se debe resaltar que el conocimiento es significativo en tanto se genere entre el investigador y los sujetos participantes en un estudio determinado, constituye, entonces un devenir dialógico el cual a través de la participación logra perfeccionar capacidades físicas y mentales. Moral (2006).

Profundizando en la concepción de la investigación como un fin al interior de la práctica educativa, se tiene que Imbernón (2002), estableció que la investigación tenía su teleología en el hecho de aportar mayor conocimiento y enriquecer las teorías existentes y que ofrecen una explicación sostenida en el tiempo a la problemática que se gesta en su unidad de análisis. El autor además, se sustrae del positivismo y propone una vuelta hacia la fenomenología defendiendo el enfoque interpretativo como medio para desarrollar la investigación pedagógica y alcanzar una continua praxis renovadora.

Otros autores por su parte son participes del desarrollo de la epistemología concibiendo a la investigación como creadora de conocimiento , pero no como resultado de mediciones y de contrastación empírica, sino como una construcción social, naturalista, cuyo génesis es el dialogo y la llegada a compromisos, de acuerdo a Dahlberg, Moss y Pence (2013) se debe generar una "política de la epistemología", contradiciendo el hecho de que el conocimiento es producto objetivo y real, sino que considera el plano intersubjetivo dándole valor a la interrelación cargada de emociones, ética y sentimientos.

Por otro lado existen defensores del enfoque cuantitativo y paradigma positivista en lo referente a la investigación pedagógica, ello como resultado del necesario conocimiento médico que debe ser inherente a una carrera en donde se cultiva la mente y el cuerpo, por ello realizar mediciones y establecer parámetros en relación a las capacidades físicas, rendimiento físico y variables asociadas es fundamental, siguiendo a Devís (1996), se tiene que lo más importe del paradigma positivista es contrastar la hipótesis empíricamente a fin de obtener resultados de orden cuantitativo, los cuales serán más fácilmente de asociar con las variables que se generan en la enseñanza, tales como: tiempo de implicación física, realimentación, control de la disciplina, entre otros (pág. 59).

A fin de dar un tratamiento teórico a la variable Aprendizaje Basado en la Investigación Kirk y Macdonald, (1998) catalogan al denominado "aprendizaje situado" subsumida dentro de enfoque constructivista pero como modelo inter- 
pretativa, es decir como parte dela tradición cualitativa lo cual es factible de ser implementado en la enseñanza de la Educación Física.

Uno de los aprendizajes situados es el que se trata en el presente estudio hace referencia a la aplicación del aprendizaje basado en investigación, el cual es útil para desarrollar habilidades en esta área, consiguiendo que, a partir de casos reales, todos los estudiantes se interesen por desarrollar investigaciones vivenciando el proceso así lo manifiestan; Rivadeneyra y Silva (2017); Peñaherrera et al., (2014); Vila et al., (2014)2014; Vizcaino, (2015)2015; Espinel et al., (2016).

\section{MÉTODOS Y MATERIALES}

Se utilizaron métodos y técnicas e instrumentos de análisis de datos cuantitativos (SPSS) con un diseño descriptivo de correlación que permitió la aplicación de dos cuestionarios, uno dirigido a la percepción del alumno de la metodología ABI y el otro al aprendizaje.

Con respecto a la pedagogía de la investigación aplicada, los resultados cuantitativos trianguladas con la técnica cualitativa de entrevistas en profundidad, estas entrevistas fueron transcritas y luego analizadas utilizando la técnica de comparación continua, para esto, se identificaron categorías y subcategorías basadas en la narrativa escrita de la entrevista, luego se formó una red conceptual, que se interpretó en base a la teoría formal.

\section{RESULTADOS}

La contrastación de la hipótesis de investigación Hi: Existiría relación directa y significativa entre la investigación pedagógica y la metodología ABI, habiéndose planteado la hipótesis nula Ho: No Existiría relación directa y significativa entre la investigación pedagógica y la metodología ABI; sometiéndose al cálculo de la correlación Rho de Spearman, se situó como alta positiva, ergo directa y significativa como se explica en los párrafos siguientes.
Al analizar los resultados obtenidos con la aplicación de los cuestionarios sobre las variables de estudio, los mismos que fueron aplicados a 91 estudiantes y 15 docentes se arribó a la conclusión de que al aplicarse la metodología del aprendizaje basado en la investigación en relación con la dimensión problematización alcanzó un valor para la Rho de Sperman $=1,00$, concluyendo que tienen una relación alta significativa, del mismo modo la dimensión, obtención de conclusiones, que marcaría la finalización de la investigación iniciada por los estudiantes bajo la conducción del docente, en el marco de la aplicación de la ABI refleja una correlación Rho de Spearman $=0,666$, un tanto más baja que la primera dimensión citada, ello se debe a que el $100 \%$ de estudiantes alcanzan plantear el problema y formularlo, pero el porcentaje disminuya conforme se va desarrollando la investigación que el estudiante planificó ejecutar.

A fin de consolidar lo expresado numéricamente en los resultados de correlación se tiene en la tabla mostrada a continuación los resultados referidos a la percepción de docente y estudiantes referidas a la aplicación de la metodología ABI.

Tabla $\mathbf{N}^{\circ} 1$. Percepción de los estudiantes y docentes sobre la aplicación de la metodología ABI.

\begin{tabular}{lcccccc}
\hline \multicolumn{1}{c}{ Nivel } & Escala & fi & hi\% & Fi & Hi\% \\
$\begin{array}{l}\text { Muy } \\
\text { Bajo }\end{array}$ & 0 & 12 & 0 & 0 & 0 & 0 \\
\hline Bajo & 13 & 25 & 0 & 0 & 0 & 0 \\
Regular & 26 & 38 & 6 & 5,7 & 6 & 5,7 \\
Alto & 39 & 51 & & 61,3 & 71 & 67 \\
\hline $\begin{array}{l}\text { Muy } \\
\text { Alto }\end{array}$ & 52 & 64 & 35 & 33 & 106 & 100 \\
& & & 106 & $100 \%$ & & \\
\hline
\end{tabular}

Fuente: Elaboración propia

Como se aprecia en la tabla No 01 un $33 \%$ de los sujetos de la muestra se ubica en la categoría muy alta referida a la percepción sobre la aplicación de la metodología ABI cuando se desarrollan las sesiones de aprendizaje, ello es consecuencia 
de la curiosidad mostrada por los estudiantes al materializar su idea de investigación, la misma que surge de su observación de la realidad, dando como producto la problematización y consiguiente formulación del problema, del mismo modo el docente al aplicar la metodología ABI percibe que el estudiante muestra interés y se siente motivado lo cual es el detonante para que prosiga con la ejecución de su proyecto de investigación.

Asimismo, la tabla siguiente contiene los resultados sobre la pedagogía aplicada en la enseñanza-aprendizaje de la investigación en las aulas de la facultad de ciencias del deporte, de acuerdo al cuestionario aplicado a docentes y estudiantes en conjunto.

Tabla $N^{\circ}$ 2. Aplicación pedagógica de la investigación

\begin{tabular}{|c|c|c|c|c|c|}
\hline Nivel & Escala & $\mathrm{Fi}$ & hi\% & $\mathrm{Fi}$ & $\mathrm{Hi} \%$ \\
\hline Muy Bajo & 012 & 0 & 0 & 0 & 0 \\
\hline Bajo & 1325 & 0 & 0 & 0 & 0 \\
\hline Regular & $26 \quad 38$ & 0 & 0 & 0 & 0 \\
\hline Alto & 3951 & 65 & 61,3 & 65 & 61,3 \\
\hline \multirow[t]{2}{*}{ Muy Alto } & 5264 & 41 & 38,7 & 106 & 100 \\
\hline & & 106 & $100 \%$ & & \\
\hline
\end{tabular}

Fuente: Elaboración propia

La pedagogía mostrada por los docentes en el desarrollo de la investigación ha sido catalogada, tanto por los docentes como por los mismos estudiantes, que participan en las sesiones de enseñanza aprendizaje como muy alta, ello se refleja en el $38,7 \%$ de sujetos de la muestra y un porcentaje de $61,3 \%$ considerado alto, consolidan este resultado.

El significado de los resultados implica que los docentes al aplicar la metodología del aprendizaje basado en la investigación obtiene buenos resultados consolidando una pedagogía adecuada en el tratamiento del tema de enseñanza aprendizaje de la investigación o metodología de la investigación para ser más preciso.
Como se expresó al iniciar la descripción y análisis de resultados mostrando los valores obtenidos con las correlaciones, se tiene la tabla No 03 que arrojó dichos resultados.

Tabla No 03. Correlación entre las dimensiones de la variable Aplicación pedagógica de la investigación y la variable utilización de la metodología ABI

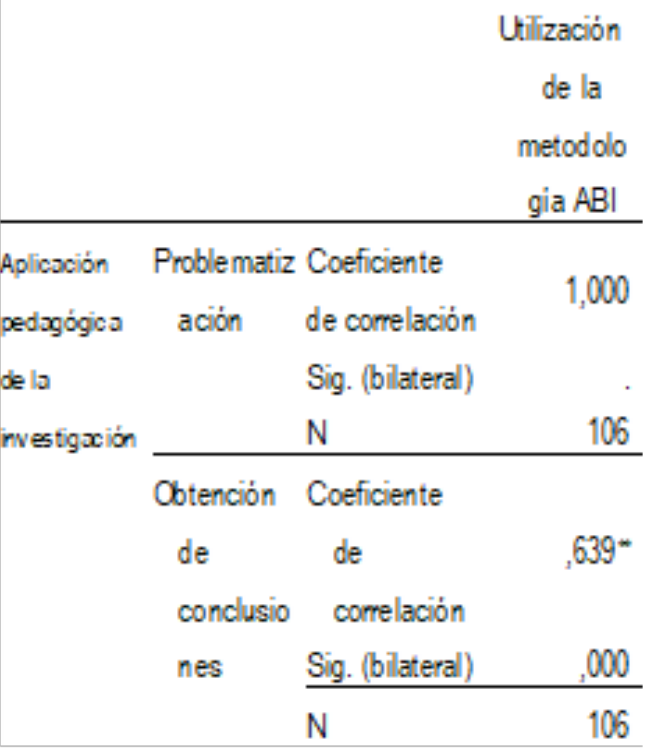

\section{DISCUSIÓN}

Además del proceso didáctico eficiente que se logra al manejar el ABI, también se observa que otro proceso que se expresa a través de la investigación es la retroalimentación, bajo su forma de entrega, que constituye el cómo se está investigando, tiene que ver con la forma en que el estudiante va construyendo las actividades de aprendizaje (deportivas) a partir de la guía y apoyo interactivo del docente.

En pleno desarrollo de evaluación formativa el estudiante expone y muestra sus saberes, es capaz de reflexionar sobre sus logros, identifica sus errores y los aprende a reconocer para mejorar su aprendizaje. La teoría sobre la evaluación formativa se sustenta en el feedback o realimentación. (permite a los profesores formadores interactuar con sus estudiantes y compartir criterios de evaluación. El estudiante identifica la brecha existente entre sus logros reales y potenciales.) 
La percepción que mostraron los estudiantes sobre esta aplicación refleja la importancia de despertar su curiosidad, el Aprendizaje Basado en la investigación constituye una herramienta heurística de vital importancia en el proceso de materializar experiencias pedagógicas relacionadas con el deporte, ya que detectado el problema, se realiza la investigación con la finalidad de comprender la realidad problemática e incluso poner en práctica una alternativa de solución critica que responda a la hipótesis o interpretación formulada, siguiendo a Mendoza (2015), se tiene que la aplicación de la metodología del ABI, constituye una forma de abordar los procesos formativos, teniendo como resultado el desarrollo del Pensamiento Crítico.

La teoría concuerda con los resultados obtenidos luego de procesar los datos recogidos de la muestra, la misma consistió en 91 estudiantes y 15 docentes obteniéndose un valor del estadístico Rho de Spearman, en relación a la aplicación de la metodología del aprendizaje basado en la investigación y la dimensión problematización, equivalente a 1,00 , lo cual de acuerdo a sus intervalo de valores corresponde a una relación alta significativa, asimismo la dimensión obtención de conclusiones como acto culminante de la investigación iniciada por los estudiantes en el marco de la aplicación de la ABI, obtuvo un valor de la Rho de Spearman de 0,666, si bien es cierto es menor que el valor obtenido con la primera dimensión, también está en el rango alto positivo y por ello aceptable.

Si se aprecia ambos valores de la Rho de Spearman se tiene que en líneas generales existe relación alta significativa entre la investigación pedagógica y la metodología $\mathrm{ABI}$ siendo aceptable la contrastación empírica.

El hecho de realizar investigaciones también implica el uso de autorregulación por parte de los estudiantes de los procesos de aprendizaje, incluye varios comportamientos, pensamientos y emociones que permiten a través del control y el análisis alcanzar metas y objetivos académicos, según Zimmerman (2000), para el caso la meta y objetivo es la contrastación de una hipótesis o la interpretación reflexiva de un contexto de modelo circular o espiral.

\section{CONCLUSIONES}

El aprendizaje basado en la investigación permite que el futuro docente de ciencias del deporte, analice su didáctica y la convierta en una nueva práctica pedagógica (praxis) acompañada de la realimentación, la misma que sustenta los procesos de evaluación y sirve como punto de partida para engendrar nuevas estrategias que permitan el desarrollo y desempeño docente siguiendo un crecimiento en espiral.

La aplicación de la metodología del aprendizaje basado en la investigación correlacionada con la dimensión problematización arrojó un resultado contundente ya que equivale al 1,00, interpretándose ello como el hecho de que la aplicación del ABI despierta una fuerte motivación en el estudiante por investigar y descubrir la solución a los problemas detectados en la realidad.

Con respecto a la correlación entre la dimensión obtención de conclusiones y la aplicación de la metodología del aprendizaje basado en la investigación obtuvo un valor para la Rho de Spearman de 0,666 , valor considerado alto significativo, lo que implica que el estudiante y el docente que participan del ABI logran recorrer todo el tramo metodológico desde el planteamiento del problema o problematización hasta la obtención de las conclusiones pasando por la prueba de hipótesis.

\section{REFERENCIAS BIBLIOGRÁFICAS}

Dahlberg , G., Moss, P., \& Pence, A. (2013). Beyond quality in early childhood education and care. New York: Routledge.: Languages of evaluation.

Devis Devis, J. (1996). Educación física, deporte y currículum: Investigación y desarrollo curricular. Madrid: Visor. Espinel, J., Robles, J., \& Ramírez, G. y. (2016). Aprendizaje Basado en la investigación. caso UNEMI. Ciencia UNEMI., 49-57. Obtenido de http://ojs.unemi.edu.ec/index. php/cienciaunemi/issue/view/34/showToc 
Imbernón, F. (2002). La investigación educativa como herramienta de formación del profesorado: reflexión y experiencias de investigación educativa. Barcelona: Graó.

Kirk, D., \& Macdonald, D. (1998). Situated Learning in Physical Education. Journal of Teaching in Physical Education,, 17, 3, 376-387.

Mendoza, P. (2015). La investigación y el desarrollo de pensamiento crítico en estudiantes universitarios. Tesis Doctoral. Universidad de Malaga, España.

Moral, C. (2006). Criterios de validez en la investigación cualitativa actual. Investigación educativa, 147-164.

Peñaherrera, M., \& Chiluiza, K. y. (2014). Inclusión del Aprendizaje Basado en Investigación (ABI) como práctica pedagógica en el diseño de programs de postgrados en Ecuador. Elaboración de unapropuesta. Journal for Educators, Teachers and Trainers, 204 -220. Obtenido de file://C:/Users/Eta/Downloads/93-361-1-PB.pdf

Rivadeneira Rodríguez, E., \& Silva Bustillos , R. (2017). Aprendizaje basado en la investigación en el trabajo autonomo y en equipo. Negotium, 5-16. Obtenido de https:// www.redalyc.org/pdf/782/78253678001.pdf

Sabino, C. (1992). El proceso de investigación. Buenos Aires: Panamericana. Obtenido de https://metodoinvestigacion.files.wordpress.com/2008/02/el-proceso-de-investigacion_carlos-sabino.pdf

Vila, R., \& Rubio, M. y. (2014). La investigación formativa a través del aprendizaje orientado a proyectos: una propuesta de innovación en el grado de pedagogía. Revista en Innovación Educativa, 24-30. Obtenido de http://www.usc. es/revistas/index.php/ie/article/view/1586/2404

Vizcaino, G. (2015). La política pública universitaria y los procesos de evaluación institucional. Algunos elementos para la reflexión. Obtenido de https://universidadsociedadec.files.wordpress.com/2015/05/la-polc3adtica-pc3bablica-universitaria-y-los-procesos-de-evaluacic $3 b 3 n$-institucional.pdf

Zimmerman, B. J. (2000). Autoeficacia: un motivo esencial para aprender. Psicología educativa contemporánea, 82-91. doi:https://doi.org/10.1006/ceps.1999.1016 